\title{
International Conference on Lymphocyte Engineering Author Index
}

\author{
Abadajos J., A10 \\ Abdo L., A4 \\ Abken H., A3 \\ Acharya D., A5 \\ Adutler-Lieber S., A13 \\ Akahori Y., A5, A6 \\ Akriv T., A12 \\ Albano L., A2 \\ Albert S., A16 \\ Alpert A., A22 \\ Alten L., A5, A22 \\ Alvanou M., A9 \\ Amaike C., A6 \\ Åmellem Ø., A17 \\ Anagnostopoulos A., A9 \\ Anakok O., A20 \\ Anderson J., A7 \\ Andresen T., A9 \\ Anguita J., A18 \\ Annoni A., A2 \\ Armstrong A., A8, A14 \\ Arndt C., A3, A4, A16 \\ Bachmann M., A3, A4, A16 \\ Bandora E. Abu, A17 \\ Barros L., A4 \\ Barzel A., A12, A19 \\ Battaglia M., A17 \\ Bednarczyk P., A21 \\ Benhar I., A12 \\ Bergmann R., A3, A4, A16 \\ Bergot A.S., A17 \\ Berndt N., A3, A4, A16 \\ Bernström K., A17 \\ Blane T., A10 \\ Boelens J.J., A11 \\ Bojin F., A7 \\ Bolsée J., A10 \\ Bonamino M., A4 \\ Bornschein S., A10, A18 \\ Boyatzis S., A14 \\ Brady J., A5 \\ Braeckmans K., A13, A20 \\ Brans T., A13 \\ Breman E., A18 \\ Brenner R., A17 \\ Brey C., A19 \\ Brey C.U., A4 \\ Bridgeman J., A14, A20 \\ Brown A., A8
}

\author{
Buchanan K., A17 \\ Buchholz C.J., A19 \\ Bujak J., A21 \\ Bulliard Y., A5 \\ Bunk S., A22 \\ Buquicchio F., A2 \\ Burton D., A10 \\ Bykova N., A11 \\ Carmi Y., A15, A16, A17 \\ carmi Y., A21 \\ Carrascosa T., A18 \\ Casares N., A6, A18 \\ Castiello M.C., A2 \\ Cheadle E., A8, A14 \\ Chicaybam L., A4 \\ Chocarro S., A18 \\ Conde-Gallastegi E., A6 \\ Coughlin Z., A5, A22 \\ Couvrette L., A10 \\ Crookes H., A20 \\ Csuka O., A7 \\ Czirbesz K., A7 \\ Danesh A., A9 \\ Dao M., A5 \\ Davies J., A17 \\ del Valle C., A18 \\ Demoulin B., A18 \\ De Smedt S.C., A20 \\ De Smedt C. S., A13 \\ Dianov D., A11 \\ Dilling T., A9 \\ Djinovic-Carugo K., A19 \\ Do M., A8 \\ Dobersberger M., A19 \\ Dosenovic P., A3 \\ Dotan I., A12, A19 \\ Dunnebach E., A11 \\ Dushek O., A4 \\ Efimov G., A11 \\ Eguizabal C., A18 \\ Eles K., A7 \\ Elizalde E., A6, A15 \\ Eshhar Z., A12 \\ Farhat-Younis L., A15, A16 \\ Farhat L., A17, A21 \\ Farkas E., A7 \\ Feldmann A., A3, A4, A16 \\ Ferreira P., A4 \\ Fontaine M., A18 \\ Fraire J., A13 \\ Frank A., A19 \\ Fried M., A12 \\ Friedman N., A13 \\ Fritsche J., A22 \\ Frosch J., A7 \\ Fujiwara H., A5, A6 \\ Fuller R., A10 \\ Gargiulo L., A7 \\ Gavriliuc O., A7 \\ Gazumyan A., A3 \\ Geiger B., A13 \\ Genovese P., A2 \\ Geoffery R., A17 \\ Gilham D., A2, A10, A14, A18 \\ Gjølberg D., A17 \\ Godeny M., A7 \\ Goetgeluk G., A13 \\ Gonzalez-Martin A., A10 \\ Gonzalez-Vaz J., A15 \\ Gorraiz M., A18 \\ Granica M., A21 \\ Gritsenko N., A12, A19 \\ Gutman H., A17 \\ Gutwillig A., A15, A16, \\ A17, A21 \\ Hamana H., A15 \\ Harris M., A17 \\ Hartmann J., A19 \\ Hartweger H., A3 \\ Hawkins R., A14, A20 \\ Hereng T.H., A17 \\ Herrera L., A18 \\ Hervas-Stubbs S., A15, A18 \\ Hervás-Stubbs S., A6 \\ Hessner M., A17 \\ Hilf N., A5 \\ Hjort M., A14 \\ Hoffmann A., A3, A4 \\ Holter W., A4, A19 \\ Honz M., A10 \\ Horn G., A12 \\ Horning M., A3 \\ Horovitz-Fried M., A19 \\ Horvath S., A7
}


Huang D., A10

Hurst N., A20

Hux JoAnn, A2

Ikeda H., A5, A6

Irvine D., A9

Jankovic M., A3

Jantz Derek, A2

Jo T., A5

Johnson Mark, A2

Johnson S., A14

Jones D., A9

Jones R.B., A9

Juan M., A18

Jureczek J., A4, A16

Jurgen K., A11

Kalaitsidou M., A14

Kalra M., A5, A22

Kasler M., A7

Kato Y., A6

Katopodi T., A14, A20

Khmelevskaya A., A11

Kilgallon A., A14

Kiryukhin D., A11

Kishi H., A15

Kittel-Boselli E., A3

Kobayashi E., A15

Koristka S., A3, A4, A16

Kosmala D., A21

Kotlan B., A7

Kotzerke J., A16

Koukoulias K., A9

Krenciute G., A8

Kueberuwa G., A8, A14, A20

Kuttruff-Coqui S., A5

Landais E., A10

Lasarte J.J., A6, A18

Leal-Ortiz S., A14

Lee Jason, A2

Lehner M., A4, A19

Lenger S., A10

Levin A. Globerson, A12

Levy D., A8

Le Brocq M., A14, A20

Li W., A10

Lieske N., A17

Liszkay G., A7

Lobner E., A4

Loureiro L., A3, A4, A16

Lozano T., A6, A18

Lo Presti V., A11

Lu B., A8

Lu T.T., A17

MacLeod D., A2

Machado H., A10

Majchrzak K., A21

Makris A., A9

Mancheño U., A6

Mancheno U., A15
Mann C., A5

Marcovecchio G., A2

Margulies C., A2

Marques L., A4

Martin-Ruiz I., A18

Martin Aaron, A2

Mata M., A5

Maurer D., A22

McCreedy Bruce, A2

McGuire A., A3

Mccann C., A9

Mercuri E., A2

Met Ö., A11

Michaux A., A10

Missel S., A5

Mitwasi N., A4

Miwa H., A5, A6

Miyahara Y., A5, A6

Mlynek G., A19

Mohamed A., A22

Mølgaard K., A11

Mota T., A9

Moya R., A17

Muflihah C. Hanif, A7

Muraguchi A., A15

Nahmad A., A12, A19

Najjar A., A12

Naldini L., A2

Narsale A., A17

Naszados G., A7

Nataf D., A12, A19

Nemazee D., A10

Nierkens S., A11

Nowicka A., A5

Nussenzweig C M., A3

Nyc A., A21

Obinger C., A19

Odé Z., A8

Økern G., A17

Okumura S., A5

Olasz J., A7

Olson A., A10

Ozawa T., A15

Pantazi C., A9

Papadopoulou A., A9

Patel A., A14

Paunescu V., A7

Pawlowski N., A5

Payne D., A17

Petersen C.T., A8

Pilunov A., A11

Pires Michelle, A2

Plantinga M., A11

Protzer U., A10

Psatha N., A9

Pühringer D., A19

Pundak-Mintz C., A12, A19

Raemdonck K., A13, A20

Raes L., A13

Raitano S., A10
Ramusino C. Cotta, A2

Rasoulouniriana D., A15, A16, A17, A21

Rehn U., A17

Reticker-Flynn N., A17

Rider P., A15, A16, A17, A21

Robertson H., A17

Romaniuk D., A11

Salzer B., A4, A19

Santana-Magal N., A15, A16, A17, A21

Santos S., A18

Saperia C., A17

Sarobe P., A15, A18

Savolt A., A7

Saye K., A10

Schaffner I., A19

Schief W., A10

Schiemann M., A10

Schiffner T., A10

Schiroli G., A2

Schöber M., A4

Schoor O., A22

Schreiber S., A10

Schrøder I.C., A17

Schüller C., A4

Schutt A., A14

Schwoebel F., A5

Seaman S M., A3

Serti E., A17

Sette A., A10

Sheetikov S., A11

Shiku H., A5, A6

Shitaoka K., A15

Shivakumar R., A5

Shmelev A., A11

Shomuradova A., A11

Sieger K., A22

Singh H., A5

Smith Jeff, A2

Sok D., A10

Sotiropoulou P., A10

Sotiropoulou A. P., A18

Spyridonidis A., A9

Stamatatos L., A3

Stamatopoulos K., A9

Stevenson E., A9

Stremersch S., A13

Suarez J., A6

Svane I.M., A11

Swoboda R., A14

Sykorova M., A14, A20

Szollar A., A7

Szopa I., A21

Takuma K., A6

Tal L., A15, A16, A17, A21

Tamayo I., A15

Tanaka Y., A5

Tawara I., A5

Taylor J J., A3

Tenuta M., A10

Thalheimer F.B., A19 
Thomas R., A17

Tran J., A10

Traxlmayr M., A19

Traxlmayr M.W., A4

Tsiolas G., A9

Tsivian L. A., A17

Uckert W., A19

Ujhelyi M., A7

Vallianou I., A9

Vandekerckhove B., A13

Van Hoeck J., A13, A20

van Til N., A11

Vavassori V., A2
Vercher E., A6

Vesga M.A., A18

Viegas M., A4

Villa A., A2

Voss J., A10

Vossfeldt H., A14

Wagner C., A22

Waks T., A12

Walter S., A5, A22

Wang L., A5, A6

Ward A., A9

Wayteck L., A20

Weinschenk T., A5, A22

Wine Y., A12, A17
Wisskirchen K., A10

Xiong R., A13, A20

Yannaki E., A9

Yiangou M., A9

Yost D., A3

Zale E., A9

Zhang H., A17

Zheng W., A8

Zielinski C., A10

Zilberstein Y., A12

Zoaby A., A12

Zogorean R., A7 